\title{
Resultados asociados con la realización de colecistectomía subtotal en un hospital de tercer nivel de Bogotá
}

\author{
Juan S. Parra P. ${ }^{1,2}$, Natalia Rodríguez R. ${ }^{1,2}$ y Camilo A. Peña M. ${ }^{1,2}$
}

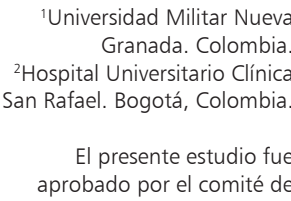
ética instituci

Recibido 2020-01-21 y aceptado 2020-05-12

Correspondencia a: Dr. Juan S. Parra P. juansebastianparra@gmail.com

\section{Results of partial cholecystectomy in a tertiary level hospital in Bogotá}

Introduction: Laparoscopic cholecystectomy is actually the gold standard surgical approach to the gallbladder disease. Some patients with severe cholecystitis and inflammatory changes that makes impossible to dissect the hepatocystic triangle. Is in this case, where the laparoscopic subtotal cholecystectomy is an option, to reduce the risk of biliary injury. Aim: To describe the clinical and surgical results in patients that underwent in laparoscopic subtotal cholecystectomy at Hospital Universitario Clínica San Rafael since February 2015 to February 2017. Materials and Method: A case series study was carried out, with a descriptive analysis to determine the distribution of them. We used contingency tables and fischer tests. Results: 28 laparoscopic subtotal cholecystectomy. The average age of 56.1 years, fifty percent was men. At the clinical characteristics, the history of diabetes and arterial hypertension was observed. There was no bile duct lesion in any and there was a reintervention due to intra-abdominal collection and only one mortality. 50\% had biliary leakage and $25 \%$ of cases required endoscopic intervention. Conclusion: Laparoscopic subtotal cholecystectomy is a safe procedure.

Key words: laparoscopic cholecystectomy; gallbladder disease; postoperative complication.

\section{Resumen}

Introducción: La colecistectomía laparoscópica es actualmente el gold standard en patología vesicular litiásica. Hay un grupo de pacientes que tiene un gran proceso inflamatorio que impide la identificación adecuada del triángulo de seguridad. Dado lo anterior, una alternativa es la realización de colecistectomía subtotal por laparoscopia, para reducir el riesgo de lesión de vía biliar. Objetivo: Describir los resultados clínicos y quirúrgicos en pacientes que se sometieron a la realización de colecistectomía subtotal laparoscópica en el Hospital Universitario Clínica San Rafael en el periodo comprendido entre febrero de 2015 y febrero de 2017. Materiales y Método: Serie de casos, donde se realizó un análisis descriptivo para determinar la distribución de las variables, se utilizaron tablas de contingencia, pruebas Fischer según el caso y su distribución en número y normalidad. Resultados: Se obtuvo información de 28 pacientes, con un promedio de edad de 56,1 años, mayor proporción de hombres. En cuanto a las características clínicas se observaron los antecedentes de diabetes e hipertensión arterial. No hubo complicaciones ni sangrado intraoperatorio importantes. En resultados posoperatorios no se presentó lesión de vía biliar en ninguno, hubo 3 reingresos $(10,7 \%)$ y hubo una reintervención relacionada con colección intraabdominal. Se reportó una mortalidad secundaria a neumonía. Conclusión: La colecistectomía subtotal es un procedimiento seguro en cuanto a la prevención de lesión de vía biliar.

Palabras clave: colecistectomía laparoscópica; colecistectomía difícil; complicaciones posquirúrgicas; colecistectomía subtotal. 


\section{Introducción}

La enfermedad litiásica biliar afecta alrededor de $1 \%$ a $4 \%$ de la población occidental cada año y de $10 \%-15 \%$ durante la vida ${ }^{1,2}$. La colecistectomía difícil es un procedimiento con aumento del riesgo quirúrgico comparado con procedimiento estándar ${ }^{3,4}$. Con incidencia de $16 \%$ en grandes series ${ }^{3}$, la colecistectomía difícil se asocia con una inflamación severa aguda o crónica, que altera la anatomía local y dificulta la disección, o con hígados cirróticos que incrementan el riesgo de sangrado. Varias técnicas de control de daños han sido propiciadas, el aumento en el uso de estas técnicas tiende a reducir la morbilidad, especialmente las lesiones de la vía biliar ${ }^{5}$. La colecistostomía descomprime las vesículas severamente inflamadas o drena el empiema, actuando como un puente para un procedimiento definitivo ${ }^{6}$. La aproximación desde fondo a bacinete es un intento para mejor identificación del triángulo de $\mathrm{Calot}^{7}$, la colecistectomía subtotal extirpa una porción de la vesícula cuando la visión de seguridad crítica no puede lograrse ${ }^{8}$. Tambien la colecistectomía subtotal ha sido asociada con fugas biliares posquirúrgicas y cálculos retenidos en el tejido remanente ${ }^{9-12}$.

\section{Materiales y Método}

Se utilizaron los registros clínicos, quirúrgicos y epidemiológicos hospitalarios de los pacientes con enfermedad litiásica de la vía biliar, se incluyeron los pacientes sometidos al procedimiento de colecistectomía subtotal por cirugía mínimamente invasiva (Figura 1) entre febrero de 2015 y febrero de 2017. La muestra fue por conveniencia y se incluyeron pacientes mayores de 18 años y con los datos completos de las variables de interés. Se rea-

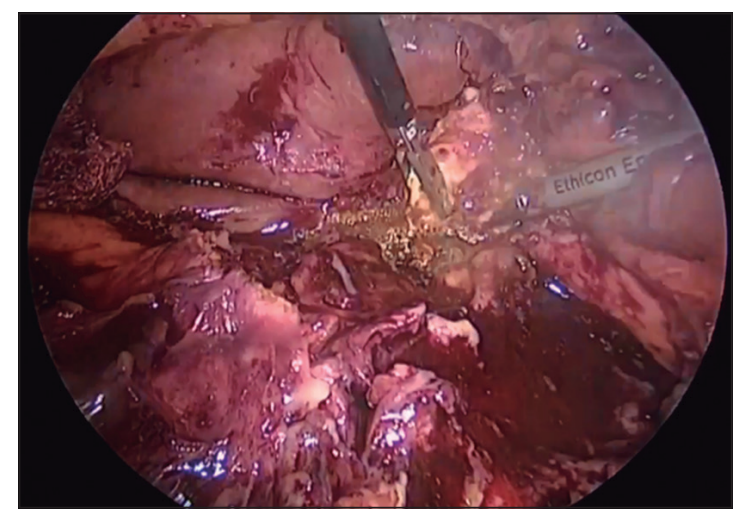

Figura 1. Colecistectomía subtotal por laparoscopia. lizó análisis descriptivo utilizando técnicas de estadística descriptiva para determinar la distribución de las variables, tablas de contingencia, pruebas Fischer según el caso y su distribución en número y normalidad.

La técnica utilizada fue disección de fondo a bacinete, resección de pared vesicular anterior, la pared posterior se electrofulguró, el muñón vesicular se dejó abierto y se colocó sistema de drenaje cerrado próximo al muñón vesicular. El retiro del dren se realizó con debito menor a $20 \mathrm{cc}$ y que no fuera bilioso, en algunos casos se daba alta a los pacientes con el dren in situ, se retiraba de manera ambulatoria.

\section{Resultados}

De un total de 1.188 colecistectomías laparoscópicas, 28 se realizaron de forma subtotal (2\%), las que se incluyeron en el estudio.

Las características según sexo y grupo de edad, así como el laboratorio, se muestran en la Tabla 1.

De las variables preoperatorias, el tiempo de evolución desde el inicio de los síntomas hasta la cirugía fue de 9,7 días, 6 pacientes habían presentado episodios de cólico biliar previos, todos de sexo masculino.

En cuanto a hallazgos ecográficos en todos los casos se reportó colelitiasis, el signo ecográfico más frecuente fue el engrosamiento de las paredes (17 pacientes), seguido de cálculo enclavado en el cuello (3 pacientes), hidrocolecisto (3 pacientes), signo de Murphy ecográfico (2 pacientes), colecistitis gangrenosa ( 2 pacientes) y líquido perivesicular (1 paciente).

Siete pacientes fueron llevados a colangiografía endoscópica (CPRE) antes de la cirugía. Se encontraron 5 coledocolitiasis, 1 estenosis que requirió inserción de stent y 2 odditis.

No se observaron complicaciones intraoperatorias.

A los 28 pacientes se les dejó un dren con un promedio de 9,2 días (DE: 6,5), con un mínimo de días de dos y el máximo de 28 . El criterio para retiro del dren fue drenaje no bilioso menor de $20 \mathrm{cc}$ al día, el paciente podía darse de alta con el dren in situ.

No hubo lesión de vía biliar y la mitad de los pacientes tuvieron fuga biliar; 1 caso presentó colección intraabdominal que requirió reintervención quirúrgica; la distribución por sexo y por categoría de edad se observa en la Figura 2.

El promedio de días hospitalarios posquirúrgico fue de 5,2 (DE:4,4) días con un mínimo de 1 día y 
Tabla 1. Características demográficas y preoperatorias

\begin{tabular}{|c|c|c|c|}
\hline & Hombre & Mujer & Total \\
\hline $\begin{array}{l}\text { Colecistectomías subtotales } \\
\text { por laparoscopia }\end{array}$ & 22 & 6 & 28 \\
\hline Edad (promedio) & 56,3 & 55,5 & 56,1 \\
\hline Comorbilidades & 6 & 2 & 8 \\
\hline Tiempo de evolución (promedio días) & 12,1 & 11 & 11,9 \\
\hline Episodios previos & 8 & 0 & 0 \\
\hline CPRE prequirúrgica & 6 & 1 & 7 \\
\hline Coledocolitiasis & 4 & 1 & 5 \\
\hline Variables de laboratorio al ingreso & Valor & $\begin{array}{c}\text { Relación } \\
\text { hombre:mujer }\end{array}$ & Total \\
\hline Leucocitos & $\begin{array}{c}<12.000 \\
12.000-18.000 \\
>=18.000\end{array}$ & $\begin{array}{l}11: 3 \\
9: 2 \\
2: 1\end{array}$ & $\begin{array}{r}14 \\
11 \\
3\end{array}$ \\
\hline Bilirrubina total (mg/dL) & $\begin{array}{c}<2 \\
>2,1\end{array}$ & $\begin{array}{r}15: 4 \\
7: 2\end{array}$ & $\begin{array}{r}19 \\
9\end{array}$ \\
\hline Bilirrubina directa $(\mathrm{mg} / \mathrm{dL})$ & $\begin{array}{l}<1 \\
>1\end{array}$ & $\begin{array}{r}15: 4 \\
7: 2\end{array}$ & $\begin{array}{r}19 \\
7\end{array}$ \\
\hline Aspartato aminotransferasa $\mathrm{u} / \mathrm{L}$ & $\begin{array}{c}<40 \\
40-120 \\
>120\end{array}$ & $\begin{array}{c}14: 3 \\
2: 2 \\
6: 1\end{array}$ & $\begin{array}{r}17 \\
4 \\
7\end{array}$ \\
\hline Alanino aminotransferasa $\mathrm{u} / \mathrm{L}$ & $\begin{array}{c}<40 \\
40-120 \\
>120\end{array}$ & $\begin{array}{c}10: 5 \\
7: 1 \\
5: 0\end{array}$ & $\begin{array}{r}15 \\
8 \\
5\end{array}$ \\
\hline Fosfatasa alcalina $(\mathrm{u} / \mathrm{L})$ & $\begin{array}{l}<141 \\
>140\end{array}$ & $\begin{array}{l}11: 4 \\
11: 1\end{array}$ & $\begin{array}{l}16 \\
12\end{array}$ \\
\hline
\end{tabular}

un máximo de 24 días. El promedio de días de incapacidad fue de 22,6 (DE:11,5) días (Figura 3), las medianas para hospitalización, tiempo posquirúrgico e incapacidad fueron de 10, 4 y 20 respectivamente.

Tres pacientes reingresaron, en los que el promedio de días de dren fue de 14 días, superior al resto de los pacientes (sin reingreso) que tuvo un promedio de 8 días, las características de los pacientes que reingresaron se muestran en la Tabla 2 . Un paciente falleció durante el seguimiento y la causa de la muerte fue insuficiencia ventilatoria secundaria a neumonía multilobar. En cuanto a los resultados de patología hubo 19 colecistitis aguda-crónica, 7 colecistitis gangrenosas y 1 colecistitis crónica folicular con ulceración. No hubo reportes de malignidad ni colecistitis xantogranulomatosa.

\section{Discusión}

La colecistectomía subtotal fue primero reportada por Madding en $1955^{13}$ para reemplazar la colecistostomía y como un procedimiento de rescate en casos donde la colecistectomía total era técnicamente difícil. Con la introducción de la colecistectomía laparoscópica por Muhe y Mouret ${ }^{14}$, esta se ha vuelto el procedimiento de elección por los resultados asociados $^{15}$. La colecistectomía subtotal laparoscópica ahora es la que más se realiza (73\%) seguida por el procedimiento abierto ${ }^{16}$.

La proporción de pacientes sometidos al procedimiento coincide con lo reportado en diferentes estudios a nivel mundial ${ }^{17,18}$. La mayoría de los pacientes
Figura 2. Distribución de complicaciones de colecistectomía subtotal laparoscópica según el sexo y por grupo de edad.

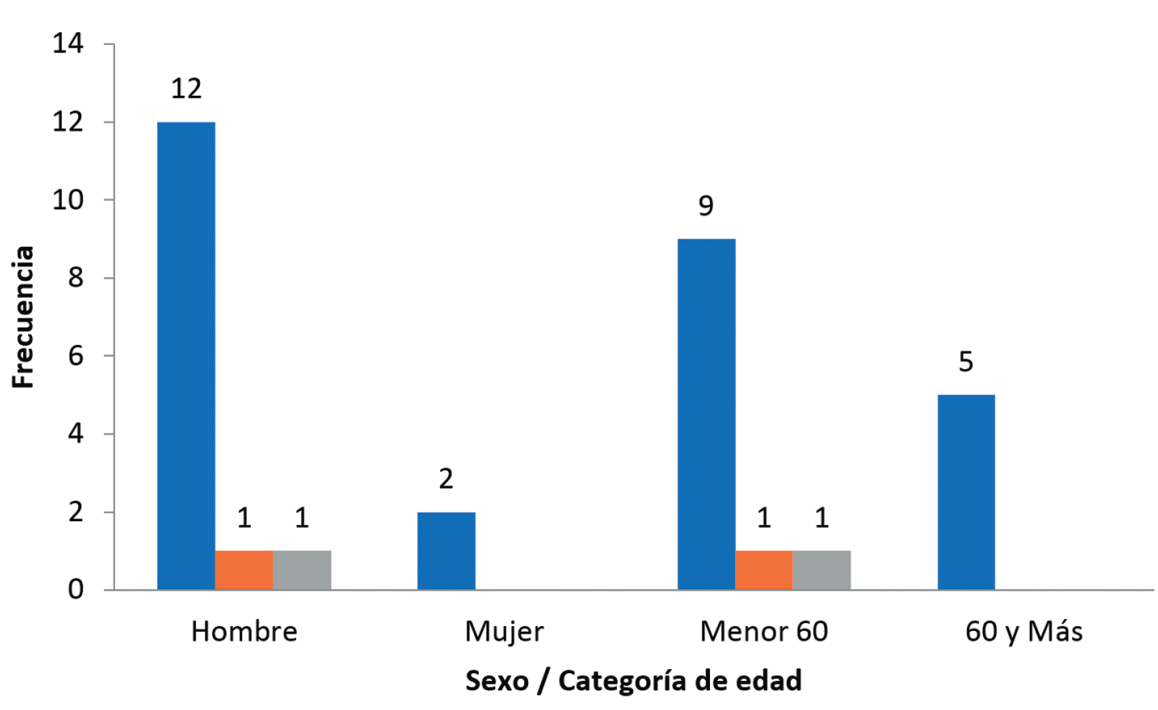

Fuga biliar

- Colección

- Mortalidad 
Tabla 2. Características de pacientes con reingreso

\begin{tabular}{|lcc|}
\hline Sexo & 1 & 33,3 \\
Hombre & 2 & 66,7 \\
Mujer & & \\
Categoría edad & 2 & 66,7 \\
Menor de 60 & 1 & 33,3 \\
60 y más & 1 & 33,3 \\
Fuga biliar & 1 & 33,3 \\
Colección & 0 & 0,0 \\
Enfermedad previa & 1 & 33,3 \\
Reintervención Qx & 3 & 100,0 \\
Endoscopia & 3 & 100 \\
\hline Total & & \\
\hline
\end{tabular}

fueron hombres con edad promedio de 56 años y con una carga de morbilidad baja, esto contrasta con lo reportado en la literatura ${ }^{18-20}$.

La mitad de los casos presentó fuga biliar, lo que está por encima de lo reportado en la literatura pese a ser un aspecto variable (5,5\% hasta $42 \%)$; ningún paciente presentó fístula biliar. Un paciente presentó colección sin requerir drenaje percutáneo lo que concuerda con lo reportado. El 25\% de los pacientes requirió CPRE lo que está por encima de lo reportado por Elshaer en un metaanálisis que incluyó más de 1.000 pacientes sometidos al procedimiento $(4,1 \%)$. Un paciente requirió reintervención quirúrgica, algo que se ha descrito en $1,8 \%$ de los estudios ${ }^{16}$

La estancia hospitalaria fue superior a lo reportado en diferentes estudios, incluida la estancia posterior al procedimiento quirúrgico ${ }^{17,19,20 .}$ El tiempo con dren fue muy superior también a lo que se reporta en los diferentes estudios ${ }^{19}$, posiblemente asociado al importante número de pacientes con fuga biliar.

Tres pacientes reingresaron, al hacer el análisis con respecto al tiempo de duración del dren posterior a cirugía, este grupo en particular mostró mayor tiempo de duración de este. Las causas de reingreso se relacionaron con patología biliar, incluyendo el paciente que presentó colección, el caso reintervenido y todos los reingresos requirieron CPRE. Estos datos son importantes, pues no se han reportado de manera sistemática en los estudios de este grupo de pacientes. Ocurrió una muerte en el seguimiento y

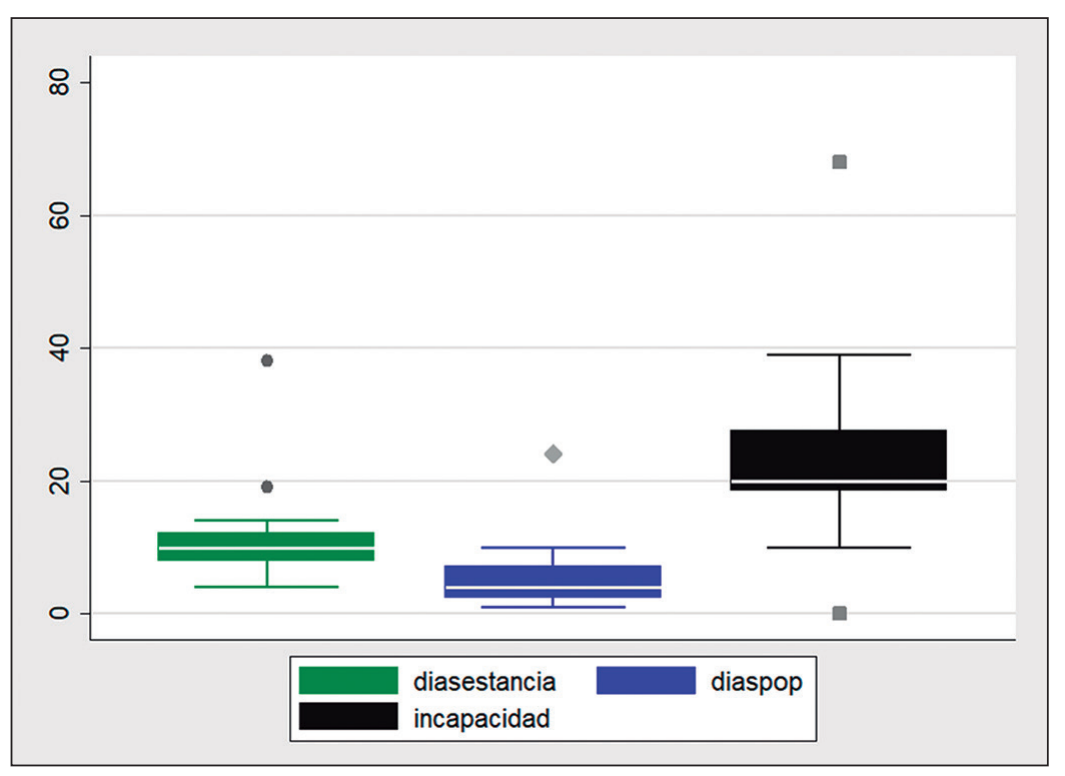

Figura 3. Distribución por días de estancia hospitalaria total, posquirúrgica e incapacidad.

se relacionó con insuficiencia ventilatoria por neumonía multilobar en un paciente con secuelas por poliomielitis. Los datos de los estudios publicados muestran tasas de mortalidad de $0,4 \%$ que generalmente se asocian a condiciones mórbidas ${ }^{16}$.

En la serie de casos más grande reportada hasta el momento, que incluyó 168 pacientes en quienes se realizó colecistectomía subtotal, la complicación más frecuente fue la colección $7 \%$ seguida por la retención de cálculos 4,2\% y no hubo mortalidad, sólo 7 pacientes $(4,1 \%)$ requirieron CPRE posoperatoria, la mayoría por coledocolitiasis residual ${ }^{20}$.

Mencionamos limitaciones del estudio: primero la naturaleza retrospectiva y descriptiva que no permite hacer asociaciones. Si bien el número de pacientes fue bajo, esto en general coincide con los estudios de centros individuales y en general se relacionan con la utilización de esta técnica que no es el manejo estándar. La exclusión de los pacientes que se sometieron a colecistectomía abierta y los que requirieron conversión desde un procedimiento inicial laparoscópico, no permite ver el comportamiento en este grupo de pacientes, además que solo se tuvo en cuenta un tipo de técnica siendo la colecistectomía fenestrada.

En general la colecistectomía laparoscópica subtotal es una herramienta importante para los cirujanos generales y hepatobiliares en situaciones complejas intraquirúrgicas que pueden derivar en alto riesgo de complicaciones posteriores. Si bien esta técnica no reemplaza a la colecistectomía total, 
los hallazgos encontrados muestran que es un procedimiento seguro, con baja tasa de complicaciones y de muerte asociadas.

\section{Responsabilidades éticas}

Protección de personas y animales. Los au- tores declaran que para esta investigación no se han realizado experimentos en seres humanos ni en animales.

Confidencialidad de los datos. Los autores declaran que en este artículo no aparecen datos de pacientes.

Conflictos de interés: no hay.

\section{Bibliografía}

1. Sanders G, Kingsnorth AN. Gallstones. BMJ 2007;335:295-9. DOI: 10.1136/ bmj.39267.452257.AD

2. Shaffer EA. Best Pract Res Clin Gastroenterol. 2006;20:981-96. doi: 10.1016/j.bpg.2006.05.004.

3. Salky BA, Edye MB. The difficult cholecystectomy: problems related to concomitant diseases. Semin Laparosc Surg. 1998;5:107-14. DOI: $10.1177 / 155335069800500205$.

4. Laws HL. The difficult cholecystectomy: problems during dissection and extraction. Semin Laparosc Surg. 1998;5:81-91. DOI: $10.1177 / 155335069800500203$.

5. Lee J, Miller P, Kermani R, Dao H, O’Donnell K. Gallbladder damage control: compromised procedure for compromised patients. Surg Endosc. 2012;26:2779-83. DOI: 10.1007/s00464012-2278-4.

6. Anderson JE, Chang DC, Talamini MA. A nationwide examination of outcomes of percutaneous cholecystostomy compared with cholecystectomy for acute cholecystitis, 1998-2010. Surg Endosc. 2013;27:3406-11. DOI: $10.1007 / \mathrm{s} 00464-$ 013-2924-5.

7. Mahmud S, Masaud M, Canna K, Nassar AHM. Fundus-first laparoscopic cholecystectomy. Surg Endosc. 2002 A;16:581-4. DOI: 10.1007/s00464-0019094-6.

8. Henneman D, da Costa DW, Vrouenraets BC, van Wagensveld BA, Lagarde SM. Laparoscopic partial cholecystectomy for the difficult gallbladder: a systematic review. Surg Endosc. 2013;27:351-8. DOI: 10.1007/s00464-012-2458-2.

9. Davis B, Castaneda G, López J. Subtotal cholecystectomy versus total cholecystectomy in complicated cholecystitis. Am Surg. 2012;78:814-7. URL: https://www.researchgate.net/profile/ Brian_Davis10/publication/228100244 Subtotal_Cholecystectomy Versus_Total_Cholecystectomy_ in_Complicated_Cholecystitis/ links/5768431908ae7f0756a224b7.pdf

10. Tian Y, Wu S-D, Su Y, Kong J, Yu H, Fan Y. Laparoscopic subtotal cholecystectomy as an alternative procedure designed to prevent bile duct injury: experience of a hospital in northern China. Surg Toda 2009;39:510-3. DOI: 10.1007/s00595-0083916-2.

11. Singhal T, Balakrishnan S, Hussain A, Nicholls J, Grandy-Smith S, El-Hasani S. Laparoscopic subtotal cholecystectomy: initial experience with laparoscopic management of difficult cholecystitis. Surg J R Coll Surg Edinb Irel. 2009;7:263-8. DOI: 10.1016/s1479-666x(09)80002-4.

12. Kuwabara J, Watanabe Y, Kameoka K, Horiuchi A, Sato K, Yukumi S, et al. Usefulness of laparoscopic subtotal cholecystectomy with operative cholangiography for severe cholecystitis. Surg Today 2014;44:462-5. DOI: $10.1007 /$ s00595-013-0626-1.

13. Madding GF. Subtotal cholecystectomy in acute cholecystitis. Am J Surg. 1955;89:604-7. DOI: 10.1016/00029610(55)90108-2.

14. Reynolds W. The first laparoscopic cholecystectomy. JSLS 2001;5:89-94.
URL: https://www.ncbi.nlm.nih.gov/pmc/ articles/PMC3015420/.

15. de Goede B, Klitsie PJ, Hagen SM, van Kempen BJH, Spronk S, Metselaar HJ, et al. Meta-analysis of laparoscopic versus open cholecystectomy for patients with liver cirrhosis and symptomatic cholecystolithiasis. Br J Surg. 2013;100:209-16. DOI: 10.1002/bjs.8911.

16. Elshaer M, Gravante G, Thomas K, Sorge R, Al-Hamali S, Ebdewi H. Subtotal cholecystectomy for "difficult gallbladders": systematic review and metaanalysis. JAMA Surg. 2015;150:159-68. DOI: 10.1001/jamasurg.2014.1219.

17. Shin M, Choi N, Yoo Y, Kim Y, Kim S, Mun S. Clinical outcomes of subtotal cholecystectomy performed for difficult cholecystectomy. Ann Surg Treat Res. 2016;91:226-32. DOI: 10.4174/ astr.2016.91.5.226.

18. Jara G, Rosciano J, Barrios W, Vegas L, Rodríguez O, Sánchez R, et al. Laparoscopic subtotal cholecystectomy: a surgical alternative to reduce complications in complex cases. Cirugia Espanola. 2017;95:465-70. DOI: 10.1016/j.ciresp.2017.07.013.

19. Supit C, Supit T, Mazni Y, Basir I. The outcome of laparoscopic subtotal cholecystectomy in difficult cases A case series. Int J Surg Case Rep. 2017;41:311-4. DOI: 10.1016/j. ijscr.2017.10.054.

20. Tay WM, Toh YJ, Shelat VG, Huey CW, Junnarkar SP, Woon W, et al. Subtotal cholecystectomy: early and long-term outcomes. Surg Endosc. 2019 Nov 06. DOI: 10.1007/s00464-019-07242-7. 